\title{
Rolling Circle Amplification (RCA)-Mediated Genome-Wide ihpRNAi Mutant Library Construction in Brassica napus
}

\author{
Shengbo Zhao ${ }^{\dagger}$, Junling Luo ${ }^{\dagger}$, Xinhua Zeng, Keqi Li, Rong Yuan, Li Zhu, Xiaofei Li, Gang Wu * \\ and Xiaohong Yan * \\ Oil Crops Research Institute of the Chinese Academy of Agricultural Sciences, Key Laboratory of Biology \\ and Genetic Improvement of Oil Crops, Ministry of Agriculture, Wuhan 430062, China; \\ shengbo_ab@163.com (S.Z.); luojunling@caas.cn (J.L.); zengxinhua@caas.cn (X.Z.); likeqi901218@163.com (K.L.); \\ yuanrong4966@163.com (R.Y.); zhuli49751@163.com (L.Z.); lixiaofei@caas.cn (X.L.) \\ * Correspondence: wugang@caas.cn (G.W.); yanxiaohong@caas.cn (X.Y.) \\ † These authors contributed equally to this work.
}

Received: 14 September 2020; Accepted: 28 September 2020; Published: 30 September 2020

\begin{abstract}
With the successful completion of genomic sequencing for Brassica napus, identification of novel genes, determination of functions performed by genes, and exploring the molecular mechanisms underlying important agronomic traits were challenged. Mutagenesis-based functional genomics techniques including chemical, physical, and insertional mutagenesis have been used successfully in the functional characterization of genes. However, these techniques had their disadvantages and inherent limitations for allopolyploid Brassica napus, which contained a large number of homologous and redundant genes. Long intron-spliced hairpin RNA (ihpRNA) constructs which contained inverted repeats of the target gene separated by an intron, had been shown to be very effective in triggering RNAi in plants. In the present study, the genome-wide long ihpRNA library of $B$. napus was constructed with the rolling circle amplification (RCA)-mediated technology. Using the phytoene desaturase (PDS) gene as a target control, it was shown that the RCA-mediated long ihpRNA construct was significantly effective in triggering gene silence in B. napus. Subsequently, the resultant long ihpRNA library was transformed into B. napus to produce corresponding RNAi mutants. Among the obtained transgenic ihpRNA population of $B$. napus, five ihpRNA lines with observable mutant phenotypes were acquired including alterations in the floral model and the stamen development. The target genes could be quickly identified using specific primers. These results showed that the RCA-mediated ihpRNA construction method was effective for the genome-wide long ihpRNA library of B. napus, therefore providing a platform for study of functional genomics in allopolyploid B. napus.
\end{abstract}

Keywords: Brassica napus; RNAi; ihpRNA; genome-wide library; mutant; functional genomics

\section{Introduction}

The complete sequencing of the genome of Brassica napus [1] has facilitated in the identification of novel genes and their corresponding functions, as well as in the exploration of the molecular mechanisms underlying important agronomic traits. Reverse genetics is an effective approach that has been utilized in functional genomic investigations in plants. Mutagenesis-based functional genomics techniques, including chemical (e.g., sodium azide or ethyl methanesulphonate), physical (e.g., c-radiation), and insertional mutagenesis (e.g., transposons or T-DNAs), have been successfully used in the functional characterization of genes [2-8]. However, these techniques have disadvantages 
and inherent limitations. For chemical or physical treatment-based mutagenesis studies, mutations are mapped or sequenced to identify mutation sites, which may be tedious and laborious in the absence of corresponding tags [9]. For insertion mutagenesis using T-DNAs or transposons, although known sequences can be utilized as DNA tags to track down flanking sequences of insertional events, it is generally difficult to generate mutants that harbor all mutations of multigene families $[10,11]$. To obtain mutants covering genome-wide genes, a large and sufficient mutant population should be produced. Physical, chemical, or insertional mutations are random and usually occur at noncoding and intergenic regions, and these imperfections make it difficult to acquire a sufficient number of mutants. In addition, phenotypes caused by recessive mutations are only manifested in $\mathrm{M}_{2}$ populations, thereby further hindering large-scale mutant analysis, and extensive labor and time are necessary to obtain a good coverage of whole genomes [9]. Most importantly, it is highly difficult to obtain mutants of essential genes because functional defects in these genes are often lethal.

B. napus (genome $A_{n} A_{n} C_{n} C_{n}$ ) is an allopolyploid that is generated by the hybridization of Brassica rapa (genome $\mathrm{A}_{\mathrm{r}} \mathrm{A}_{\mathrm{r}}$ ) and Brassica oleracea (genome $\mathrm{C}_{\mathrm{o}} \mathrm{C}_{\mathrm{o}}$ ), followed by chromosome doubling [12,13]. Furthermore, the genomes of $B$. oleracea and B. rapa share high similarity in nucleotide sequences [14]. Together with more ancient polyploidizations, the genome of $B$. napus has thus accrued a large number of homologous and redundant genes [15]. The B. napus genome assembly also contains numerous noncoding sequences, in which $34.8 \%$ are transposable elements. RNA sequencing has shown that the genome of $B$. napus possesses about 101,040 gene models, including 91,167 genes that have been confirmed through matches using B. oleracea and/or B. rapa predicted proteomes [15]. These findings also suggest that the application of the above-mentioned mutagenesis approaches to $B$. napus at the genome-level scale is highly tedious and laborious.

RNA interference (RNAi), also known as post-transcriptional gene silencing (PTGS), which is based on the ability to generate double-stranded RNA (dsRNA), causes targeted knockdown of endogenous genes. RNAi-mediated basic mechanisms of target gene silencing are conserved across kingdoms. dsRNAs are initially processed into 20-24 nt small interfering RNA (siRNA) by the RNase III-like enzyme Dicer [16,17]. The resulting siRNAs are then integrated into RNA-induced silencing complexes (RISCs), which subsequently results in the degradation of target mRNAs by interacting with homologous mRNAs [18]. Compared to insertional/physical/chemical mutagenesis approaches, the RNAi strategy offers the ability to specifically target selected genes, members of gene families and redundant genes, members of a given pathway, and essential genes that are induced by promoters $[19,20]$ and to produce graded levels of knockdowns and knockouts [21]. By these approaches, RNAi offers the flexibility needed in determining gene functions.

RNAi has been successfully utilized as an effective approach in improving disease resistance and food quality of crops [21-24]. Long intron-spliced hairpin RNA (ihpRNA) constructs that contain homologous antisense and sense inverted repeats of target genes spaced by an intron have been shown to be highly effective in inducing RNAi in plants, with a silencing frequency of $>70 \%[21,24,25]$. The rolling circle amplification (RCA)-mediated ihpRNA construction technology facilitates in the efficient and inexpensive construction of genome-wide ihpRNA libraries [26,27]. In a typical RCA, a circular template is isothermally amplified by Phi29 DNA polymerase at $30{ }^{\circ} \mathrm{C}$, generating strands averaging $70 \mathrm{~kb}$ in length, which contain a large number of tandem repeats of the starting molecule. These strands in turn serve as templates for new syntheses, thus amplification enters an exponential phase, in which the rate of initiation of new strands is proportional to the quantity of the starting DNA $[28,29]$. Therefore, RNAi transgenic populations may be generated using RCA-mediated long ihpRNAi library construction that targets all transcripts in plants.

In the present study, the long ihpRNA library of B. napus was constructed using the RCA approach. Using the phytoene desaturase (PDS) gene as a control target, we determined that RCA-mediated long ihpRNA constructs effectively trigger gene silencing in B. napus. Subsequently, the resulting long ihpRNA library was transformed into B. napus to produce a RNAi mutant library at a genome-wide scale. Among the obtained transgenic ihpRNA population of $B$. napus, the target genes could be quickly 
identified using specific primers, and five ihpRNA lines with observable mutant phenotypes were acquired including alterations in the floral model and the stamen development. These results showed that the RCA-mediated ihpRNA construction method was effective for the genome-wide long ihpRNA library of $B$. napus, therefore providing a platform for functional studies of genes on a genome-wide scale in B. napus.

\section{Results}

\subsection{RCA-Mediated ihpRNA Library Construction Procedure}

The procedure in generating high-throughput ihpRNA is outlined in Figure 1. The improved intermediate cloning vector Pkan-BsaI was highly efficient for cDNA cloning, containing the ccdB lethal gene and facilitating in the insertion of double strand cDNAs (dscDNAs) (Step 1; Figure 1). DNA insertions were digested, leading to asymmetrical 4-nt 5' overhangs 'ACCT' and 'TCCC', respectively, at the two ends, which avoided self-ligation of DNA fragments and tandem ligation between fragments (Step 2; Figure 1). Two different synthetic adaptors (adaptor 1 and adaptor 2) contained long self-complementary stretches that are respectively capable of forming a hairpin-loop structure by self-annealing. Two different sticky ends were introduced into the adaptors. A 80-nt spliceable intronic sequence derived from $B n M S 5^{a}$ [30] of B. napus was located in loop2 of adaptor 2 , serving as a spacer between the antisense and sense sequences of the resulting inverted repeats, stabilizing the hpRNA DNA clones in bacteria, and triggering RNAi effects in transgenic B. napus plants (Step 3; Figure 1). The ligation products among DNA fragments, adaptor 1, and adaptor 2, formed a dumbbell-like structure with a single-stranded closed circle (Step 3; Figure 1). RCA was performed to yield a tandem array of double-stranded inverted repeat DNA units by employing the loop2-specific primer pair loop2-F and loop2-R and Phi29 DNA polymerase (Step 4; Figure 1). RCA products were digested and ligated to the eukaryotic expression vector $\mathrm{pBI} 121$, generating the final ihpRNA library expression constructs (Step 5; Figure 1).

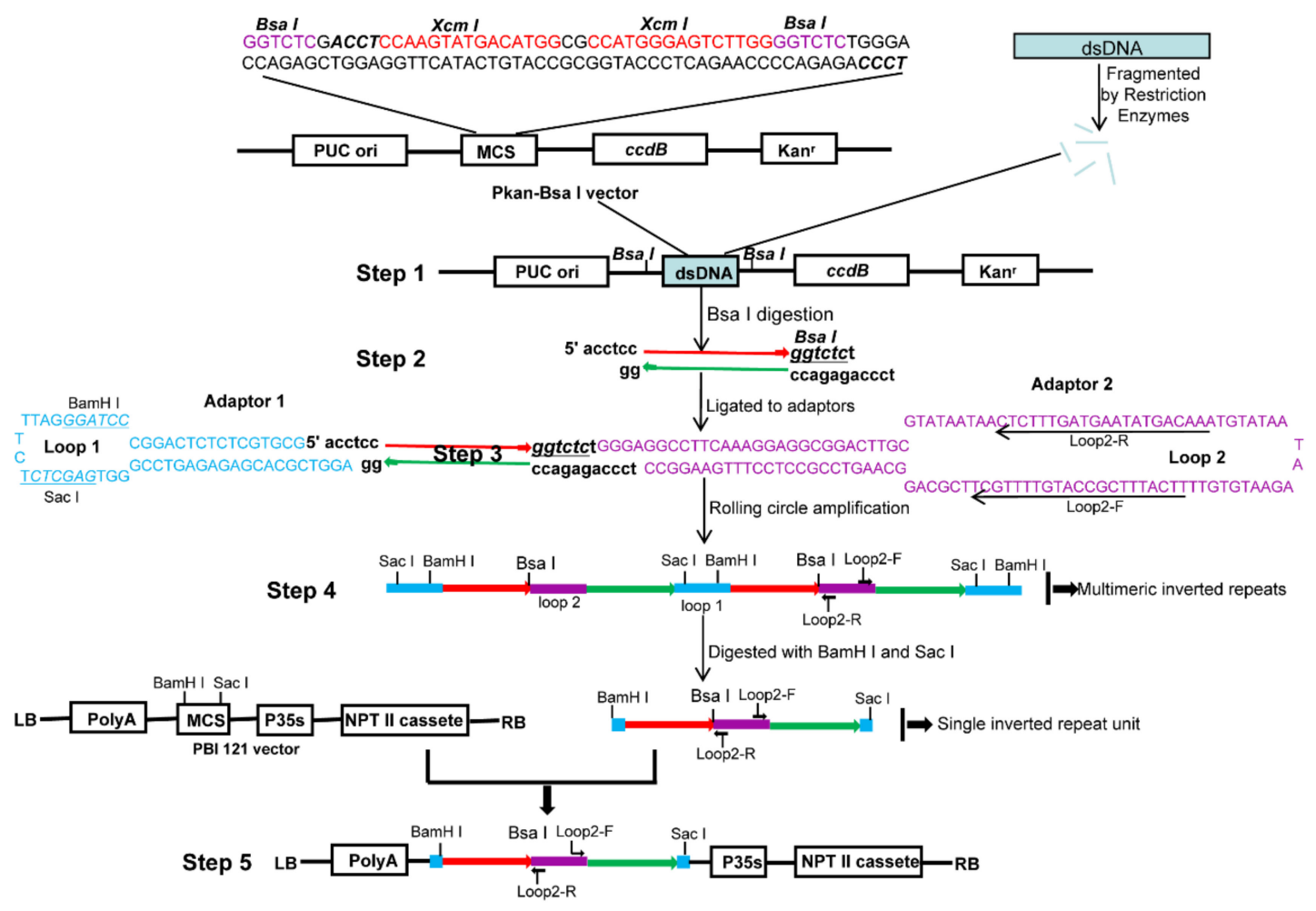

Figure 1. The procedure for RCA-mediated ihpRNA library construction. (Step 1) The Pkan-BsaI vector contains two $B s a$ I sites that were used in generating two different sticky termini in the DNA insertional 
fragments, two XcmI sites for generating the T-ended vector, and the $c c d B$ gene for avoiding the empty vector during the intermediate cDNA library construction process. dsDNAs were fragmented with RsaI, BsaI, BamHI, SacI, BglII, and HindIII restriction enzymes, blunted with T4 DNA polymerase, 'A' ends were added and ligated to pKan-BsaI. (Step 2) DNA insertion fragments were excised using BsaI, generating BsaI-digested fragments with two asymmetrical sticky termini. (Step 3) The fragments were connected to adaptors 1 and 2 through dissymmetrical sticky ends. Two adaptors carried correspondingly sticky ends complementary to BsaI-digested fragments. (Step 4) RCA was performed to yield a tandem array of double-stranded inverted repeat DNA units by employing the loop2-specific primer pair loop2-F and loop2-R and Phi29 DNA polymerase. (Step 5) RCA products were digested with $B a m \mathrm{HI}$ and $\mathrm{SacI}$, and the digested fragments were ligated to the eukaryotic expression vector pBI121, generating the final ihpRNA library expression construction. The light blue rectangle is fragmented dsDNA. Blue bases and lines are the adaptor1 sequence. Purple bases and lines are the adaptor2 sequence. Red line with the arrow is the sense strand of dsDNA. Green line with the arrow is the anti-sense strand of dsDNA.

\subsection{RCA-Mediated Construction of PDS ihpRNA}

To investigate whether RCA effectively generates iphRNA constructs, the B. napus phytoene desaturase (PDS) gene coding sequence of $450 \mathrm{bp}$ in size (from the initial codon ATG) was amplified using the primer pair PDS-F1/PDS-R1 (Figure 2A), an 'A' was added and inserted into the pKan-BsaI using the T-A approach. Following $B s a$ I digestion, PDS fragments with two different sticky ends, respectively, were obtained (Figure 2A), which then were ligated to adaptor 1 and adaptor 2, forming closed circular DNA molecules. Agarose gel electrophoresis of the ligation product showed that almost all of the BsaI-digested PDS DNA fragments were connected to both adaptors, generating adaptor1:PDS DNA::adaptor2 closed circular ligation products (Figure 2B). RCA were performed using the ligation products, Phi29, and the loop2-specific primer pair loop2-F/loop2-R. Large DNA molecules containing inverted repeat units of double-stranded DNA were observed (Figure 2C). The RCA products were confirmed by BamHI and SacI digestion, which generated 1.1-kb of linear fragment that was approximately two-fold greater in size than the unamplified PDS control, and by a further digestion for the 1.1-kb fragment with $B s a \mathrm{I}$, which produced a 500-bp fragment, including the PDS control and partial sequences of loop1, and a 600-bp fragment, including 450-bp PDS control fragment and 128-bp adaptor 2(Figure 2D). These results indicated that a pool of single inverted repeat units for PDS were formed by RCA. Finally, BamHI- and SacI-digested fragments of RCA products were inserted into eukaryotic expression vector pBI121, generating a 121-PDS-ihpRNA construct (Figure 2E).

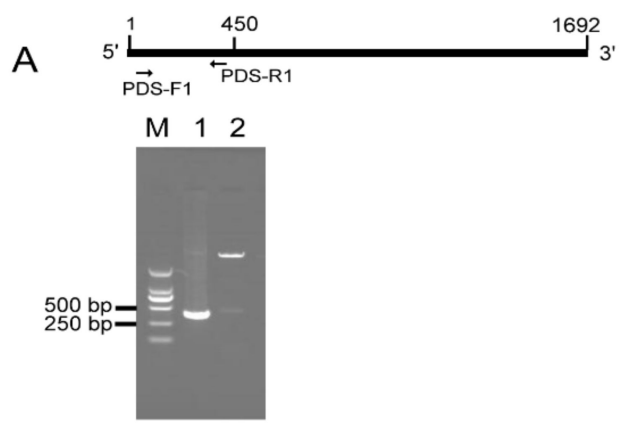

Figure 2. Cont. 

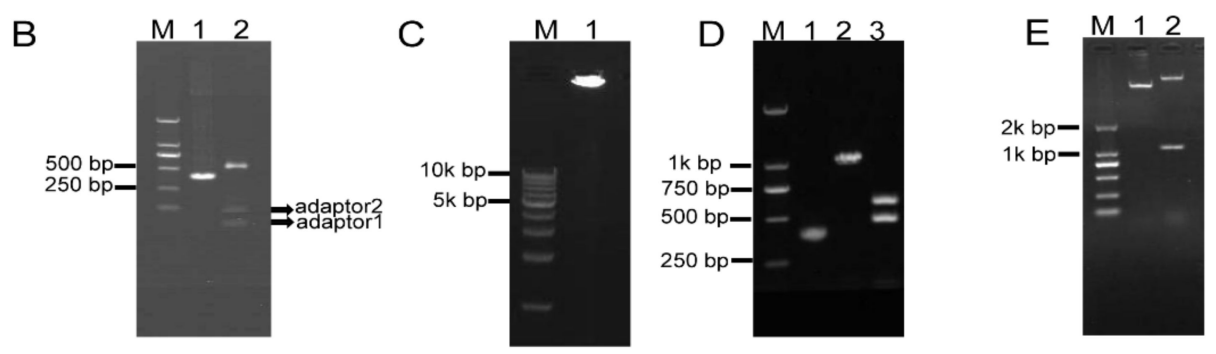

Figure 2. RCA-mediated construction of PDS ihpRNA. (A) Amplification and cloning into pKan-BsaI. A 400-bp PDS fragment was amplified using the PDS-F and PDS-R primers (lane 1), followed by T-A cloning. BsaI digestion indicates that a 450-bp PDS fragment was inserted into the pKAN-BsaI vector (lane 2). (B) Formation of closed circular molecules. BsaI-digested PDS fragment is shown in lane 1, ligation products between two adaptors and the BsaI-digested PDS fragment are shown in the upper band in lane 2, and free adaptors are shown in the lower bands in lane 2. (C) Rolling circular amplification. Closed circular DNA was amplified using Phi29 DNA polymerase and loop2-specific primers loop2-F and loop2-R, resulting in large double DNA molecules containing multiple-unit inverted repeats (lane 1). (D) Verification of inverted DNA repeats. The PDS control DNA is shown in lane 1. RCA products were digested with Bam HI and SacI, generating a 1.1-kbp fragment in lane 2. BamHI and SacI-digested fragments were further cut using BsaI, generating a 500-bp fragment and a 600-bp fragment (lane 3). (E) Construction of the pBI121-PDS-ihpRNA. BamHI and SacI-digested PDS fragment of the RCA products was inserted into eukaryotic expression vector pBI121, generating the PDS-ihpRNA. The construct was confirmed by BamHI and SacI digestion (lane 1).

\subsection{Testing of Eukaryotic Expression 121-PDS-ihpRNA Construct}

The 121-PDS-ihpRNA eukaryotic expressional construct was initially transferred into Agrobacterium strain GV3101 by electroporation, which was then transformed into B. napus cultivar zhongshuang 6 [31] via Agrobacterium-mediated gene transformation [32] to generate transgenic PDS-ihpRNA plants. Currently, this method of genetic transformation is considered to be of high efficiency (about 17\%) [33]. The binary vector pBI121 harbors the neomycin phosphotransferase gene (NPTII) resistance selection marker (Figure 3A). The selection marker-resistant regenerated $\mathrm{T}_{0}$ transformants were initially subjected to kanamycin-based selection and then rooted well in selective medium. The selected resistant $\mathrm{T}_{0}$ transformants were then verified by PCR-based screening using the corresponding primer pairs NPTII-F and NPTII-R, which were specific for the NPTII gene in the binary construct (Figure 3A). A total of five transgenic plants (approximately 17\% transformation efficiency) were obtained, all displaying photo-bleaching phenotypic characteristics (Figure 3B).

Other research has shown that Small RNAs are implicated in post-transcriptional RNA silencing in transgenic plants. RNAs transcribed in RNA silencing transgenic plants form dsRNAs that trigger siRNA biogenesis, thus causing RNA silencing in transgenic plants [34]. In addition, hpRNA by the 35S Pol II promoter can result in the accumulation of predominantly 21-nt siRNAs [35-38]. To verify whether the PDS gene was silenced, qRT-PCR corresponding to the PDS target gene in five RNAi $\mathrm{T}_{0}$ lines was investigated, which confirmed that the mRNA expression level of the target PDS had significantly decreased (Figure $3 C$ ). These results suggested that the PDS ihpRNA construct effectively silenced the PDS target gene.

Due to the complex secondary hairpin structures formed by ihpRNA constructs, special primers (avoiding specificity to the stem of the hairpin structure) were designed to efficiently amplify the hpRNA target sequence in the binary $\mathrm{pBI} 21$ vector. The primer pairs loop2-F and 121-F or loop2-R and 121-R were used to identify the target gene sequences of the ihpRNA lines (Figure 3D). For the five PDS RNAi lines, loop2-F and 121-F or loop2-R and 121-R primer pair successfully amplified bands of the desired size, and the amplification products were sequenced, demonstrating that the PDS ihpRNA expression cassette was successfully integrated into the B. napus plants (Figure 3D). This result indicated 
that these two pairs of primers could be employed to rapidly identify the candidate target genes of the ihpRNA lines.

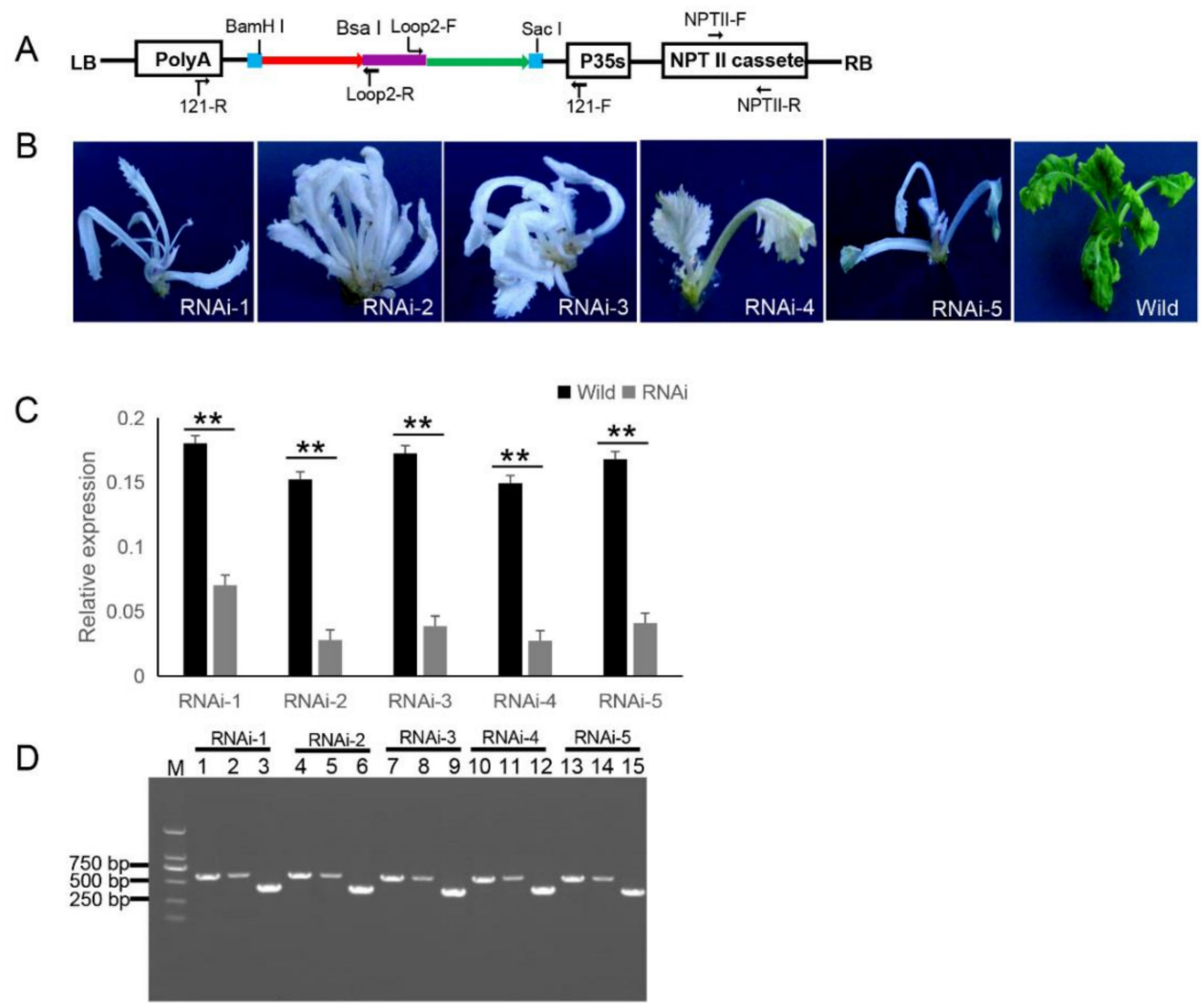

Figure 3. 121-PDS-ihpRNA-mediated silencing of the PDS gene. (A) Map of the binary vector 121-PDS-ihpRNA. NPTII-F and NPTII-R primers were used in the identification of positive transgenic plants, the primer pairs loop2-F/121-F and loop2-R/121-R were utilized to rapidly screen target genes. Blue line is the adaptor1 sequence. Purple line is the adaptor2 sequence. Red line with the arrow is the sense strand of dsDNA. Green line with the arrow is the anti-sense strand of dsDNA. (B) Interfering phenotypes of 5 PDS-ihpRNA transgenic plants. RNAi-1, RNAi-2, RNAi-3, RNAi-4, and RNAi-5 were PDS-ihpRNA transgenic plants. Wild was the transgenic negative plant. (C) qRT-PCR analysis of the PDS target gene mRNA level in the five hpRNA lines shown in (B) using the primer pair PDS-F2/PDS-R2. Data shown as mean \pm s.d., ${ }^{* *}, p<0.05$.. (D) Integration into the Brassica napus genome for the PDS ihpRNA expression cassette. For the five PDS RNAi lines, loop2-F and 121-F or loop2-R and 121-R primer pair could amplify the bands with the desired size, demonstrating that the PDS ihpRNA expression cassette was successfully integrated into the B. napus plants. Lanes 3, 6, 9, 12, and 15 show the 450-bp PDS control; lanes 1, 4, 7, 10, and 13 exhibit the PCR products of loop2-F and 121-F; lanes 2, 5, 8, 11, and 14 depict the PCR products of loop2-R and 121-R.

\subsection{Construction of the Intermediate B. napus cDNA Library}

To investigate most of the B. napus transcripts, total RNA was extracted from various tissues at different developmental stages (see Section 4), including roots, stems, leaves, inflorescences, and siliques. The high-quality, first-strand, full-length cDNAs were synthesized using the SMARTer PCR cDNA synthesis kit (Clontech Laboratories Inc., Mountain View, CA, USA) (Figure 4A). Double-stranded cDNA (dscDNA) was obtained by amplification of the first-strand cDNA templates using LD PCR [39]. For LD PCR, overcycled dsDNA resulted in less representative transcripts, whereas undercycling generates lower dsDNA yield. Therefore, a series of cycles, including 15, 18, 21, 24, and 27 cycles, were performed to optimize the number of PCR cycles. Figure $4 \mathrm{~B}$ shows that the number of LD PCR products reached a plateau at 24 cycles. After 24 cycles, a high-molecular weight smear was detected in 
the gel, demonstrating that LD PCR was overcycled. Therefore, the optimal number of PCR cycles was 24 (Figure 4B). The dsDNAs were subsequently fragmented using restriction enzymes, and fragments of 200-800 bp in size were gel-fractionated (Figure 4C) and ligated into pKan-BsaI using the T-vector ligation strategy, yielding the intermediate cDNA library that contained $9.2 \times 10^{6}$ clones. About $100 \%$ of the clones contained insertional fragments of $200-800 \mathrm{bp}$ in size (Figure 4D). These results indicated that the intermediate library was of high quality, with a relatively low empty-vector rate and was suitable for subsequent ihpRNA library construction.
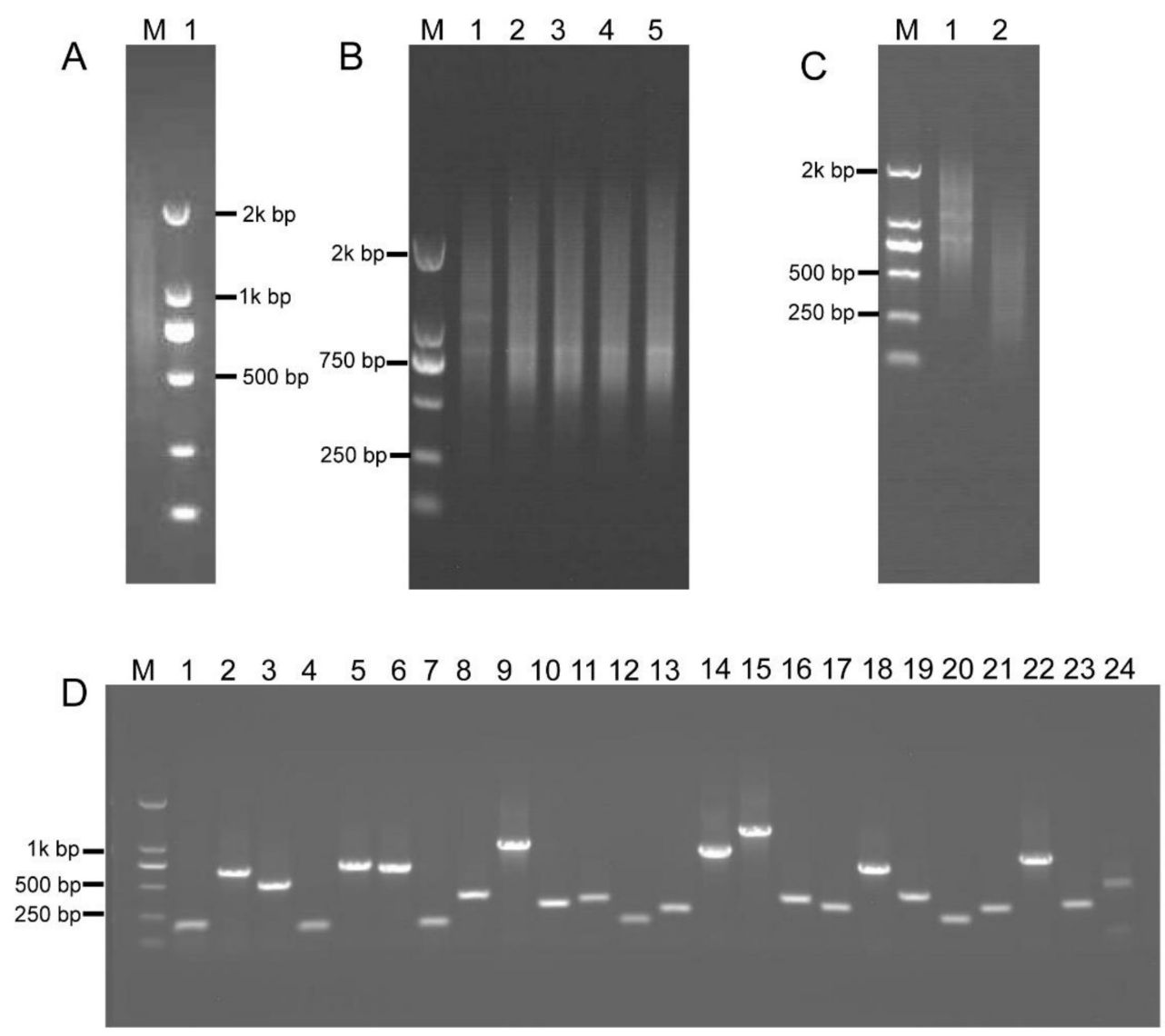

Figure 4. Construction of the intermediate B. napus cDNA library. (A) First-strand cDNA was synthesized (lane 1). (B) Optimization of the conditions for LD-PCR. A range of PCR cycles was performed. Lanes 1-5 correspond to 15, 18, 21, 24, and 27 cycles, respectively. (C) dsDNAs were fragmented with restriction enzymes, RsaI, BsaI, BamHI, SacI, BglII, and Hind III, and fragments 200-800 bp in size were gel-fractionated (lane 2), lane 1 shows dsDNA before digestion. (D) Random PCR of inserts in the intermediate cDNA library. Approximately $100 \%$ of the clones contained inserts ranging from 200 to $800 \mathrm{bp}$ in size (lanes 1-24).

\subsection{RCA-Induced ihpRNA Library Construction of B. napus}

$B s a I$-digested intermediate cDNA library insertional fragments with two different $B s a$ adhesive termini were obtained from plasmid DNA of the intermediate library and were ligated with adaptors 1 and 2, thereby generating closed circular DNA molecules (Figure 5A). RCA was performed using circular DNAs and loop2-specific primers loop2-F and loop2-R. To confirm that RCA yielded linear concatamers of inverted repeat DNAs, the RCA products were analyzed by restriction enzyme digestion. Figure 5B shows that double digestion with Bam HI and SacI generated fragments within the expected size range of 400-1600 bp and containing a pool of inverted repeat units, whose size was approximately double that of the unamplified BsaI-digested cDNA control. Fragments digested with BamHI and SacI were then ligated into the binary pBI121 vector. Ligation products were introduced into Escherichia 
coli $\mathrm{DH} 5 \alpha$ cells by electroporation, thereby generating an ihpRNA library consisting of $7.9 \times 10^{6}$ clones. To examine whether insertions in the ihpRNA library contain inverted repeats of diverse target sequences, 23 clones were randomly selected and analyzed by PCR amplification with primer pair loop2-F and 121-F, and almost all of these contained insertional inverted repeats DNAs (Figure 5C).

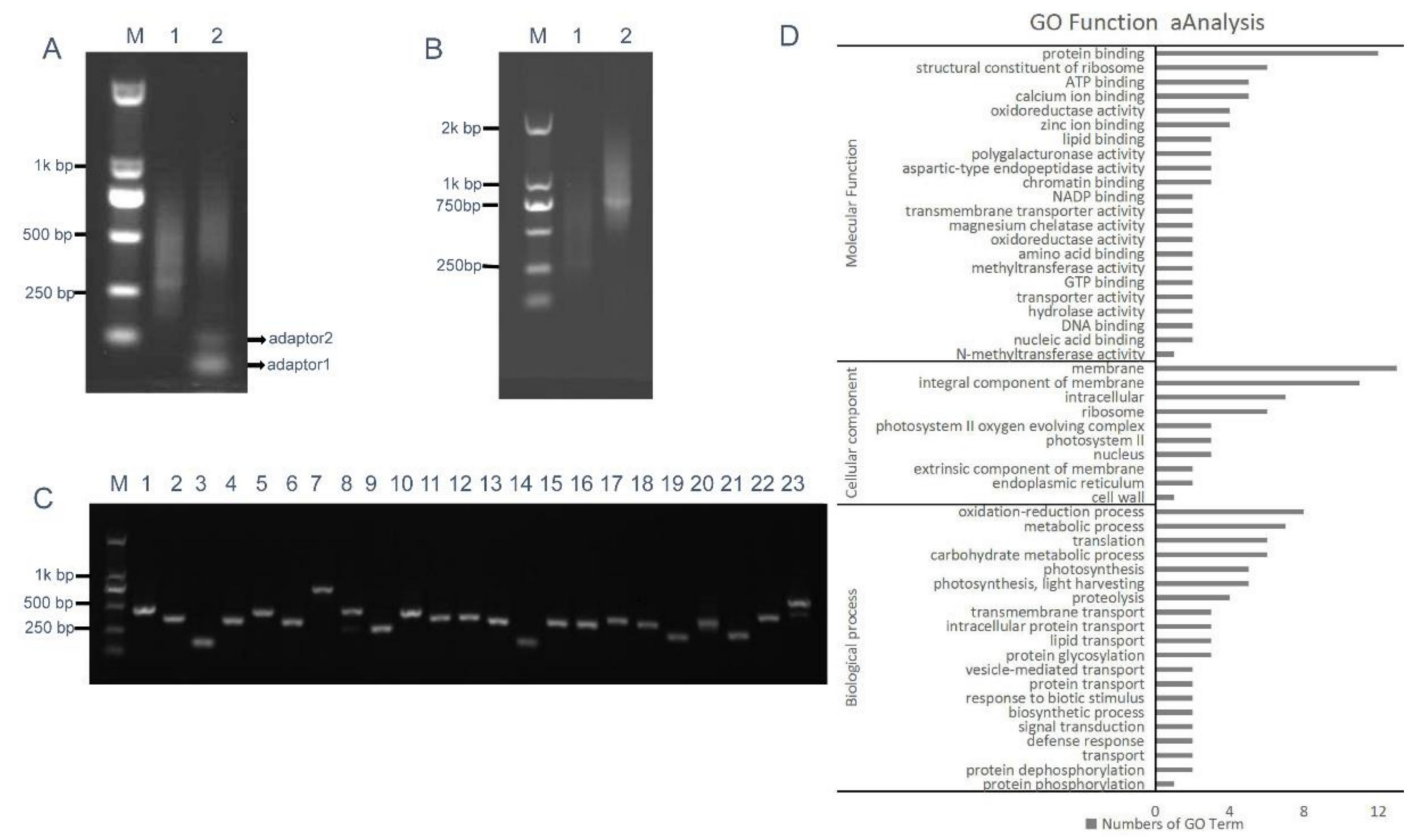

Figure 5. RCA-mediated ihpRNA mutant library construction of B. napus. (A) The intermediate cDNA library was digested with $B s a \mathrm{I}$, generating DNA fragments of 200-700 bp in size with two different $B s a I$ adhesive termini (lane 1), and purified BsaI-digested cDNA library insertional fragments were ligated with adaptors 1 and 2, leading to closed circular DNA molecules (lane 2). (B) The RCA products were digested with $\mathrm{BamHI}$ and $\mathrm{SacI}$ to release single-unit inverted repeats. DNA fragments of 400-1400 bp, representing the single unit inverted repeats (lane 2), were approximately double that of the unamplified BsaI-digested cDNA control (lane 2) in size. (C) Random PCR of inserts in the binary pBI121. Approximately 100\% of the clones contained inserts ranging from 200 to $800 \mathrm{bp}$ in size (lanes 1-23). (D) Histogram showing Gene Ontology functional analysis of randomly selected insertional fragments. The frequency of GO terms was analyzed using GO Slim Assignment. The y-axis and x-axis indicate the names of clusters and the number of genes in each cluster, respectively.

We further investigated the quality of the ihpRNA library by sequencing a subset of randomly selected clones using loop2-F and loop2-R primers and analyzing sequence data against $B$. napus sequence databases. Of the 200 clones from the ihpRNA library, 192 (96\%) had an inverted repeat sequence of $>100 \mathrm{bp}$ in length (average size: $300 \mathrm{bp}$ ), corresponding to 187 genes or gene families (Table S2). These genes represent a variety of functional categories, including cellular component, biological process, and molecular function (Figure 5D). This finding indicated that the constructed ihpRNA library was of high quality and with a good level of gene coverage.

\subsection{Generation of the Transgenic ihpRNA Mutant Population of B. napus}

For transformation of $B$. napus, the ihpRNA library was initially introduced into A. tumefaciens GV3101 by electroporation, thereby generating the corresponding Agrobacterium library that contained at least $8.4 \times 10^{6}$ clones. The library was then utilized to transform cultivar Zhongshuang 6 to generate a transgenic ihpRNA population of B. napus. Among the 96 positive $\mathrm{T}_{0}$ transgenic lines, five ihpRNA lines with observable mutant phenotype, including the abnormal floral model (Figure 6A), male sterility (Figure 6B), smooth leaves (Figure 6C), curly leaves (Figure 6D), and yellowed leaves (Figure 6E), were acquired. The mutants in flower organs, including alterations in the floral model 
(Figure 6A) and the stamen development (Figure 6B) were named library-RNAi1 and library-RNAi2, respectively. The target gene sequences of library-RNAi1 and library-RNAi2 lines were easily identified by amplification of the ihpRNA sequence using the 121-R/Loop2-R or 121-F/Loop2-F primer pairs and sequenced, showing that the ihpRNA expression cassette was inserted into the genome of B. napus (Figure S1). The target gene of library-RNAi1 was one of BnAP2 family members, which was essential for the determination of the identity of sepals and petals and was a primary member of class A genes, containing three copies in B.napus genome (LOC106411443, LOC106445506, and LOC106445506) [32,40]. The hpRNA in library-RNAi1 line matched perfectly with the sequence of LOC106411443 from 11 to $170 \mathrm{nt}$. The target gene of library-RNAi2 was one of BnMS5 family members, which was involved in fertility-related traits in B. napus [30,41,42]. BnMS5 gene family members targeted by the hpRNA in library-RNAi2 line included LOC106453110, LOC111208847, LOC106453110, LOC106411501, and LOC106360299. The hpRNA in library-RNAi2 line matched perfectly with the sequence of LOC111208847 from 17-376 nt. As the relative nucleotide sequence identities of the targeted gene members in library-RNAi1 and library-RNAi2 were more than $90 \%$, respectively, primers were designed according to conserved sequences (Table S1). qRT-PCR of the target genes in the two RNAi $\mathrm{T}_{0}$ lines were examined, which confirmed that the expression level of the target mRNA significantly decreased, indicating that the hpRNA derived from ihpRNA library construction induced efficient silencing of itself and the related family members (Figure 6F). For the other three mutants, the target sequences needed to be analyzed on the $T_{1}$ or $T_{2}$ generation due to the presence of multiple transgene insertion sites. These findings showed that our ihpRNAi mutant library may potentially be utilized as a powerful tool for gene identification in B. napus.
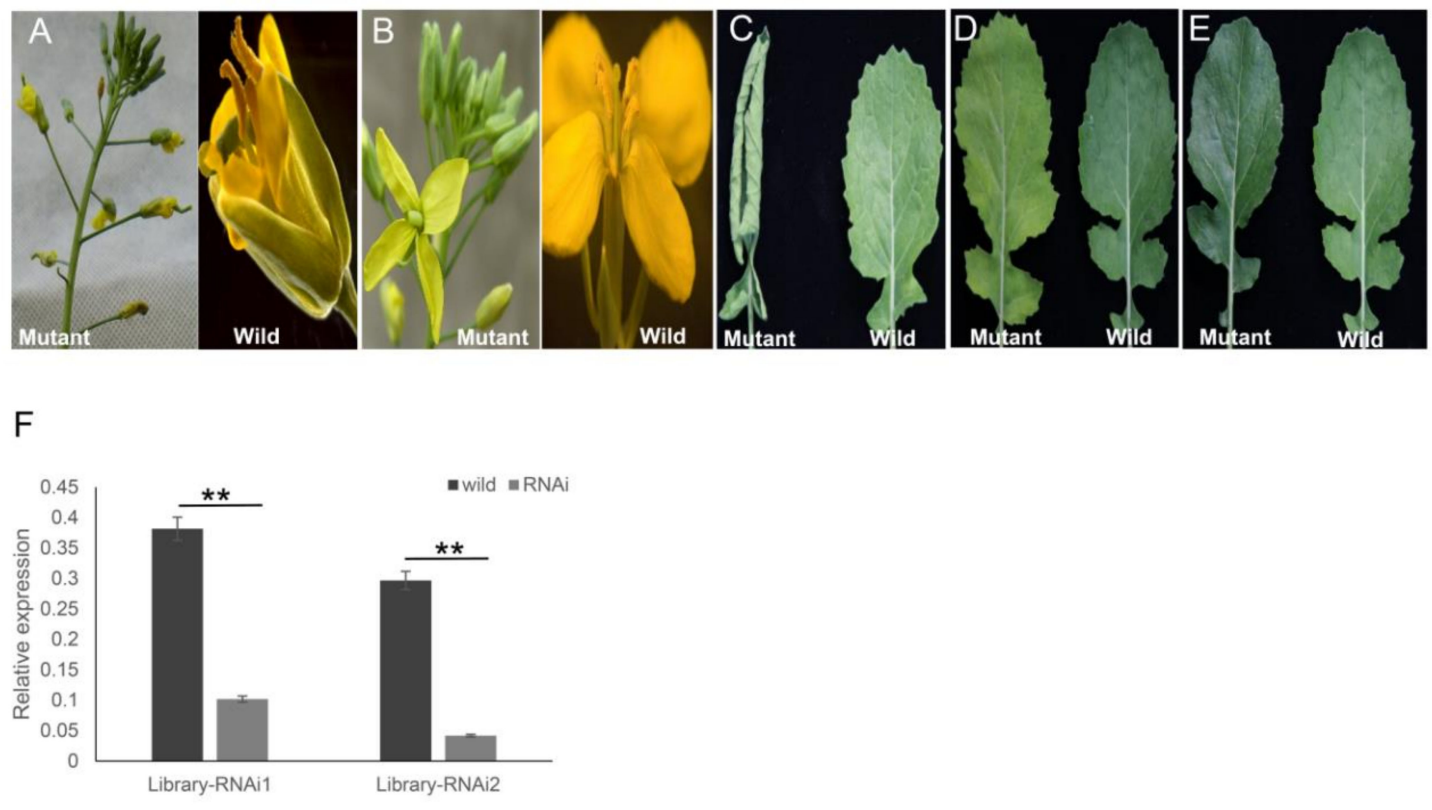

Figure 6. Five representative mutant phenotypes of the ihpRNA transgenic oilseed rape. (A) Alterations in the floral model. (B) Alterations in the stamen development. (C) Curly leaves. (D) Yellowed leaves. (E) Smooth leaves. (F) qRT-PCR analysis of target gene mRNA level in the two ihpRNA lines shown in (A) and (B). Data shown as mean \pm s.d., ${ }^{* *}, p<0.05$.

\section{Discussion}

Various ihpRNA constructs employing conventional cloning systems have been extensively employed in functional studies for gene silencing [24]. However, traditional ihpRNA vector construction methods involve specific PCR amplification of target sequences and single or multistep ligation reactions, which were generally tedious and expensive. High-throughput gene silencing, which simultaneously involves the construction of a large number of ihpRNA vectors targeting thousands of genes in a specific 
plant species, thus remain as a major challenge $[28,43]$. The RCA strategy provides an effective method of producing an array of tandem template copies, thereby allowing the simultaneous formation of a large number of ihpRNA constructs. Using RCA, long ihpRNA libraries of Arabidopsis and rice have been successfully generated [26,27]. In the present study, to improve the efficiency of the RCA system, bidirectional PCR using two loop-specific primers were designed, thereby eliminating noninverted repeats that often result from the use of six-nucleotide random primers $[28,43]$.

The present study selected 200-800 bp of dsDNA fragments for hpRNA construction. We avoided using longer dsDNA fragments because a previous report has shown that inverted DNA repeats with a relatively short spacer and very long stems are unstable in bacteria [44]. In addition, studies have demonstrated that approximately $120 \mathrm{bp}$ fragments could induce efficient gene silence [21,44]. In addition, ihpRNA constructs spliced by introns were more effective in triggering gene silencing in plants [24]. Therefore, a $90 \mathrm{bp}$ intron as the spacer of stems was introduced to generate a B. napus ihpRNA library. Furthermore, the intron-specific primer combined with the primer flanking the binary vector could quickly screen candidate target genes or gene families. Using this improved RCA and ihpRNA strategy, we produced a long ihpRNA library with good coverage of B. napus genes.

Approximately 3.5\% of EMS-mutagenized or T-DNA insertion populations, which generally harbor mutations occur in nonexonic sequences, display visible mutant phenotypes [45], whereas $50 \%$ of positive ihpRNA transgenic lines of a rice ihpRNA library targeting exonic sequences exhibit visible phenotypes [27]. For tetraploid B. napus, its genome possesses a large number of homologous genes. Simultaneous multigene repression is unlikely to occur through T-DNA or EMS mutagenesis in oilseed rape. The present study generated an ihpRNA construct library from a cDNA library that targeted exonic sequences, thereby providing a cost-effective way of silencing all or most homologous genes or gene family members in B. napus. However, previous reports have indicated that detecting conditional or subtle mutant phenotypes is generally difficult [46,47]. The efficiency of detecting invisible mutants largely depends upon how closely or under what conditions these mutants are being investigated. No visible mutant phenotypes were far from confirmation that a gene was nonfunctional or redundant. Several approaches may be utilized to screen for B. napus mutant phenotypes, which includes the development of standards of normal development and growth during various growth stages and establishing a set of growth conditions to ease detection of conditional mutant phenotypes.

The ihpRNA library constructs used in this study were driven by a strong constitutive promoter, namely, the CaMV $35 \mathrm{~S}$ promoter. Alternatively, an inducible or tissue-specific promoter may be utilized in future studies to explore the functions of housekeeping or essential gene functions in future studied genes. In addition, ihpRNA-induced RNAi often knocks down target genes, rather than knocking out gene expression, which restricts its application of gene function requiring only a low level of mRNA accumulation. Moreover, ihpRNAi mutant libraries largely depend on genetic transformation and tissue culture, which can cause somaclonal variations, thereby generating confusing ihpRNA-induced mutant phenotypes. These problems could be resolved by investigating cosegregation between mutant phenotypes and targeting genes in the segregating transgenic population. As RNAi is a homology dependent process, another major drawback of gene silencing is that it suppresses unintended genes which are similar in sequence to the targeted silencing genes, but have an ectopic phenotype. This issue can be figured out by selection of a unique region of the target sequence.

\section{Materials and Methods}

\subsection{Construction of the $p K A N-B s a I$ Vector and Cloning of the PDS Gene Fragment}

Two oligonucleotides with $5^{\prime}$ phosphorylation were synthesized, including the sense strand pKan- $B s a(+)$ and the antisense strand pKan-Bsa (-), as shown in Table S1. Two BsaI sites flanking $X \mathrm{cmI}$ sites were introduced into the two oligonucleotides. The two oligonucleotides were used in the amplification of the pKAN vector, and the amplification products were self-ligated to form the intermediate vector pKan-BsaI. The improved intermediate cloning vector Pkan-BsaI contained two 
XcmI sites, two BsaI sites flanking each XcmI site, and the ccdB lethal gene. XcmI digestion generated two 3'-T overhangs, transforming pKan-BsaI into a T-ended vector that would facilitate the insertion of double strand cDNAs (dscDNAs). The lethal $c c d B$ gene could avoid the recovery of $E$. coli clones with empty vectors. The recognition sequence of $B s a \mathrm{I}$ is GGTCTC(N) $)_{1-5}$, and the two $B s a I$ sites within the pKan-BsaI vector were 'GGTCTCGACCT' and 'GGTCTCTGGGA'.

The 450-bp coding sequence of the PDS gene was isolated from cDNA of B. napus cultivar Zhongshuang 6 (Oil Crops Research Institute, CAAS, Wuhan, China) using high fidelity Pfu, to which an ' $\mathrm{A}$ ' was attached. The PDS fragment was separated in a $1.5 \%$ agarose gel, excised, and purified, and then inserted into the pKan-BsaI vector using the T-A cloning strategy. The ligation products were then transformed into Escherichia coli DB3.1, and the resultant colonies were randomly selected and sequenced.

\subsection{B. napus Intermediate cDNA Library Construction}

B. napus Zhongshuang 6 plants were planted under normal farming conditions in the field of the Oil Crops Research Institute of the Chinese Academy of Agricultural Sciences in Hubei, China. Various tissues at different developmental stages were collected, namely, roots, stems, and leaves at the seedling stage; inflorescences at the flowering stage; and siliques at 10,15, 20, 25 and 30 days after pollination. Total RNA was extracted from these tissues, respectively, using TRIzol ${ }^{\circledR}$ (Invitrogen, Carlsbad, CA, USA) and mixed at equal amounts. cDNA was synthesized using the SMARTer PCR cDNA synthesis Kit according to the SMART cDNA library manual (Clontech Laboratories Inc., Mountain View, CA, USA). dsDNA products of long distance PCR were digested using RsaI, BglII, HindIII, BamHI, SacI and BsaI, resulting in fragments $200-800$ bp in length, and then treated using T4 DNA polymerase to generating blunt termini. ' $A$ ' tails were attached to the 3 ' termini using Taq DNA polymerase. The DNA fragments were then purified and ligated to a pKan-BsaI vector using the T-A strategy, generating an intermediate cDNA library that consisted of a total of $9.2 \times 10^{6}$ clones.

\subsection{The Rolling Circle Amplification (RCA)}

Two oligonucleotides with 5' phosphorylation, namely, adaptor 1 and adaptor 2, were synthesized, as shown in Table S1. The oligos were annealed as previously described [48], forming the stem-loop structure. Adaptor 1 carried a 5' overhang 'AGGT' that was complementary to the 'ACCT' terminus of BsaI-digested DNA insertion fragments, whereas adaptor 2 harbored a 5' overhang 'GGGA' end that was complementary to the 'TCCC' terminus of the digested DNA fragments. BamHI and SacI sites were introduced into the loop of adaptor 1 , which was convenient for subsequent connection of the inverted repeat units to the eukaryotic expression vector.

For generation of closed circular DNA molecules, the insertional fragments were excised from the pKan-BsaI vector by $B s a \mathrm{I}$ digestion followed by gel purification, and about $400 \mathrm{ng}$ of the purified DNA fragments was mixed with $200 \mathrm{ng}$ each of adaptor 1 and adaptor 2, $3 \mu \mathrm{L}$ of T4 DNA ligase (NEB, England), and $3 \mu \mathrm{L}$ of ligation buffer in a total reaction volume of $30 \mu \mathrm{L}$. Following overnight incubation at $16{ }^{\circ} \mathrm{C}$, the ligation products, namely, closed circular DNAs, were purified and dissolved in $100 \mu \mathrm{L}$ of sterile water.

For RCA, $30 \mu \mathrm{L}$ of the ligation reaction products was mixed with $10 \mu \mathrm{L}$ of Phi29 buffer and $5 \mu \mathrm{L}$ each of $10 \mu \mathrm{M}$ loop2-specific primers, namely, loop2-F and loop2-R (Table S1). The mixture was heated at $95{ }^{\circ} \mathrm{C}$ for $5 \mathrm{~min}$ and chilled on ice for $15 \mathrm{~min}$, to which $5 \mu \mathrm{L}$ of $10 \mathrm{mM}$ dNTPs, and $2 \mu \mathrm{L}$ of Phi29 polymerase (Fermentas, Lithuania) were added. After $6-8 \mathrm{~h}$ of incubation at $30^{\circ} \mathrm{C}$, the amplified DNAs were precipitated with 0.1 volume of $3 \mathrm{M}$ sodium acetate and 2.5 volumes of ethanol, and dissolved in $40 \mu \mathrm{L}$ of sterile water.

\section{4. ihpRNA Library Construction}

To generate closed circular cDNAs, the intermediate cDNA library was fragmented with BsaI, and the insertion dsDNA fragments were gel-purified and ligated to adaptor 1 and adaptor 2 . 
The closed circular cDNAs were amplified by RCA using loop2-specific primers (loop2-F and loop2-R), yielding linear concatamers of inverted repeat DNAs. The RCA products were digested using BamHI and $\mathrm{SacI}$ and then ligated into the binary vector $\mathrm{pBI} 121$. The ligation products were transformed into Agrobacterium tumefaciens strain GV3101 by electroporation, generating an ihpRNA library consisting of $7.9 \times 10^{6}$ clones.

\subsection{B. napus Transformation}

Agrobacterium-induced transformation of B. napus was conducted as described elsewhere [49]. Kanamycin-resistant plantlets were planted in a plant growth room. The growth conditions were $20 \pm$ $2{ }^{\circ} \mathrm{C}$ under a $16 \mathrm{~h} / 8 \mathrm{~h}$ photoperiod at a light intensity of $44 \mu \mathrm{mol} \mathrm{m}{ }^{-2} \cdot \mathrm{s}^{-1}$ and $60-90 \%$ relative humidity. Kanamycin-positive transformants were further verified by PCR using primers specific to the NptII gene (Npt-F, Npt-R). The primers used in amplification are listed in Table S1.

\subsection{Quantitative Real-Time PCR ( $q R T-P C R)$}

The extracted total RNA was initially treated with DNaseI to remove any genomic DNA contamination and then used as template for cDNA synthesis with a reverse transcription kit (Promega Corp., WI, USA) according to the manufacturer's recommendations. qRT-PCR was performed using an Applied Biosystems Prism 7500 Analyzer and GoTaq qPCR Master Mix (Promega Corp., WI, USA). Three independent biological and technical replicates were used for each plant. The results were analyzed using the $2^{-\Delta \Delta C T}$ method [50]. The actin gene of B. napus was used as internal control for normalization. The primers used are listed in Table S1.

\subsection{Gene Ontology (GO) Function Analysis of Randomly Selected Genes}

GO is a usual standardized gene analysis classification system which categorizes genes and gene products in terms of their biological process, cellular component, and molecular function. Gene Ontology (GO) is an international standardized gene functional classification system that describes properties of genes and their products in any organism, containing three ontologies: molecular function, cellular component, and biological process. A total of 99 genes from the ihpRNA library belonging to 52 categories were found (Table S3). These genes were involved in biochemistry, metabolism, development, and so on.

Supplementary Materials: Supplementary materials can be found at http://www.mdpi.com/1422-0067/21/19/ 7243/s1. Figure S1: The ihpRNA sequences of library-RNAi1 and library-RNAi2. Blue bases are vector sequences used for rapid identificaion of target genes. Red and green bases are inverted repeats of target genes. Purple bases are the intron sequence. Table S1: DNA oligonucleotide sequences; Table S2: 187 genes of randomly selected clones from the ihpRNA library; Table S3: GO terms of 99 genes from the ihpRNA library.

Author Contributions: Conceptualization, X.Y.; Data curation, S.Z., K.L. and X.L.; Formal analysis, L.Z.; Funding acquisition, X.Y.; Investigation, J.L.; Methodology, S.Z., X.Z. and R.Y.; Supervision, G.W.; Writing-original draft, S.Z. and J.L.; Writing - review and editing, X.Y. All authors have read and agreed to the published version of the manuscript.

Funding: This research was funded by grants from the National Natural Science Foundation of China (grant number 31671733), the National Grand Project of Science and Technology (grant number 2018ZX0801105B), the Breeding Special Grants of Seven Major Crops (2017YFD0102000), and the Major Research Project of CAAS Science and Technology Innovation.

Conflicts of Interest: The authors declare no conflict of interest. 


\section{Abbreviations}

$\begin{array}{ll}\text { ihpRNA } & \text { Long intron-spliced hairpin RNA } \\ \text { RCA } & \text { Rolling circle amplifica } \\ \text { RNAi } & \text { RNA interference } \\ \text { PTGS } & \text { Post-transcriptional gene silencing } \\ \text { dsRNA } & \text { Double-stranded RNA } \\ \text { siRNA } & \text { Small interfering RNA } \\ \text { RISCs } & \text { RNA-induced silencing complexes } \\ \text { PDS } & \text { Phytoene desaturase }\end{array}$

\section{References}

1. Wang, X.; Wang, H.; Wang, J.; Sun, R.; Wu, J.; Liu, S.; Bai, Y.; Mun, J.H.; Bancroft, I.; Cheng, F.; et al. The genome of the mesopolyploid crop species Brassica rapa. Nat. Genet. 2011, 43, 1035-1039. [CrossRef]

2. Ahn, J.H.; Kim, J.; Yoo, S.J.; Yoo, S.Y.; Roh, H.; Choi, J.H.; Choi, M.S.; Chung, K.S.; Han, E.J.; Hong, S.M.; et al. Isolation of 151 mutants that have developmental defects from T-DNA tagging. Plant Cell Physiol. 2007, 48, 169-178. [CrossRef]

3. Bolle, C.; Schneider, A.; Leister, D. Perspectives on systematic analyses of gene function in Arabidopsis thaliana: New tools, topics and trends. Curr. Genom. 2011, 12, 1-14. [CrossRef] [PubMed]

4. Chang, Y.; Long, T.; Wu, C. Effort and contribution of T-DNA insertion mutant library for rice functional genomics research in China: Review and perspective. J. Integr. Plant Biol. 2012, 54, 953-966. [CrossRef]

5. Kolesnik, T.; Szeverenyi, I.; Bachmann, D.; Kumar, C.S.; Jiang, S.; Ramamoorthy, R.; Cai, M.; Ma, Z.G.; Sundaresan, V.; Ramachandran, S. Establishing an efficient Ac/Ds tagging system in rice: Large-scale analysis of Ds flanking sequences. Plant J. 2004, 37, 301-314. [CrossRef] [PubMed]

6. Piffanelli, P.; Droc, G.; Mieulet, D.; Lanau, N.; Bes, M.; Bourgeois, E.; Rouviere, C.; Gavory, F.; Cruaud, C.; Ghesquiere, A.; et al. Large-scale characterization of Tos17 insertion sites in a rice T-DNA mutant library. Plant Mol. Biol. 2007, 65, 587-601. [CrossRef] [PubMed]

7. Till, B.J.; Cooper, J.; Tai, T.H.; Colowit, P.; Greene, E.A.; Henikoff, S.; Comai, L. Discovery of chemically induced mutations in rice by TILLING. BMC Plant Biol. 2007, 7, 19. [CrossRef] [PubMed]

8. Wang, N.; Long, T.; Yao, W.; Xiong, L.; Zhang, Q.; Wu, C. Mutant resources for the functional analysis of the rice genome. Mol. Plant 2013, 6, 596-604. [CrossRef]

9. Matthew, L. RNAi for plant functional genomics. Comp. Funct. Genom. 2004, 5, 240-244. [CrossRef]

10. Kim, S.K. http://C.Elegans: Mining the functional genomic landscape. Nature Rev. Genet. 2001, 2, 681-689. [CrossRef]

11. Stracke, R.; Werber, M.; Weisshaar, B. The R2R3-MYB gene family in Arabidopsis thaliana. Curr. Opin. Plant Biol. 2001, 4, 447-456. [CrossRef]

12. Johnston, J.S.; Pepper, A.E.; Hall, A.E.; Chen, Z.J.; Hodnett, G.; Drabek, J.; Lopez, R.; Price, H.J. Evolution of genome size in Brassicaceae. Ann. Bot. 2005, 95, 229-235. [CrossRef] [PubMed]

13. Yogeeswaran, K.; Frary, A.; York, T.L.; Amenta, A.; Lesser, A.H.; Nasrallah, J.B.; Tanksley, S.D.; Nasrallah, M.E. Comparative genome analyses of Arabidopsis spp.: Inferring chromosomal rearrangement events in the evolutionary history of A. thaliana. Genome Res. 2005, 15, 505-515. [CrossRef] [PubMed]

14. Liu, S.; Liu, Y.; Yang, X.; Tong, C.; Edwards, D.; Parkin, I.A.; Zhao, M.; Ma, J.; Yu, J.; Huang, S.; et al. The Brassica oleracea genome reveals the asymmetrical evolution of polyploid genomes. Nat. Commun. 2014, 5, 3930. [CrossRef] [PubMed]

15. Chalhoub, B.; Denoeud, F.; Liu, S.; Parkin, I.A.; Tang, H.; Wang, X.; Chiquet, J.; Belcram, H.; Tong, C.; Samans, B.; et al. Plant genetics. Early allopolyploid evolution in the post-Neolithic Brassica napus oilseed genome. Science 2014, 345, 950-953. [CrossRef]

16. Axtell, M.J. Classification and comparison of small RNAs from plants. Annu. Rev. Plant Biol. 2013, 64, 137-159. [CrossRef]

17. Wang, M.B.; Masuta, C.; Smith, N.A.; Shimura, H. RNA silencing and plant viral diseases. Mol. Plant Microbe Interact. 2012, 25, 1275-1285. [CrossRef]

18. Jones-Rhoades, M.W.; Bartel, D.P.; Bartel, B. MicroRNAs and their regulatory roles in plants. Annu. Rev. Plant Biol. 2006, 57, 19-53. [CrossRef] 
19. Chen, S.; Hofius, D.; Sonnewald, U.; Börnke, F. Temporal and spatial control of gene silencing in transgenic plants by inducible expression of double-stranded RNA. Plant J. 2003, 36, 731-740. [CrossRef]

20. Guo, H.S.; Fei, J.F.; Xie, Q.; Chua, N.H. A chemical-regulated inducible RNAi system in plants. Plant J. 2003, 34, 383-392. [CrossRef]

21. Wesley, S.V.; Helliwell, C.A.; Smith, N.A.; Wang, M.B.; Rouse, D.T.; Liu, Q.; Gooding, P.S.; Singh, S.P.; Abbott, D.; Stoutjesdijk, P.A.; et al. Construct design for efficient, effective and high-throughput gene silencing in plants. Plant J. 2001, 27, 581-590. [CrossRef] [PubMed]

22. Helliwell, C.A.; Waterhouse, P.M. Constructs and methods for hairpin RNA-mediated gene silencing in plants. Methods Enzymol. 2005, 392, 24-35. [PubMed]

23. Mansoor, S.; Amin, I.; Hussain, M.; Zafar, Y.; Briddon, R.W. Engineering novel traits in plants through RNA interference. Trends Plant Sci. 2006, 11, 559-565. [CrossRef]

24. Smith, N.A.; Singh, S.P.; Wang, M.B.; Stoutjesdijk, P.A.; Green, A.G.; Waterhouse, P.M. Total silencing by intron-spliced hairpin RNAs. Nature 2000, 407, 319-320. [CrossRef] [PubMed]

25. Waterhouse, P.M.; Helliwell, C.A. Exploring plant genomes by RNA-induced gene silencing. Nat. Rev. Genet. 2003, 4, 29-38. [CrossRef] [PubMed]

26. Wang, L.; Fan, Y.L. Rolling circle amplification-mediated long hairpin RNA library construction in plants. Methods Mol Biol. 2012, 894, 309-321.

27. Wang, L.; Zheng, J.; Luo, Y.; Xu, T.; Zhang, Q.; Zhang, L.; Xu, M.; Wan, J.; Wang, M.B.; Zhang, C.; et al. Construction of a genomewide RNAi mutant library in rice. Plant Biotechnol. J. 2013, 11, 997-1005. [CrossRef]

28. Dean, F.B.; Nelson, J.R.; Giesler, T.L.; Lasken, R.S. Rapid amplification of plasmid and phage DNA using Phi29 DNA polymerase and multiply-primed rolling circle amplification. Genome Res. 2001, 11, 1095-1099. [CrossRef]

29. Hutchison, C.A.; Smith, H.O.; Pfannkoch, C.; Venter, J.C. Cell-free cloning using phi29 DNA polymerase. Proc. Natl. Acad. Sci. USA 2005, 102, 17332-17336. [CrossRef]

30. Zeng, X.; Yan, X.; Yuan, R.; Li, K.; Wu, Y.; Liu, F.; Luo, J.; Li, J.; Wu, G. Identification and analysis of MS5d: A gene that affects double-strand break (DSB) repair during meiosis I in Brassica napus microsporocytes. Front. Plant Sci. 2016, 7, 1966. [CrossRef]

31. Zou, C.; Li, G.; Qu, Z.; Chen, D.; Cheng, Y.; Zheng, P. Breeding of Brassica napus cultivar Zhongshuang No. 6 with double-low, higher-yield and resistance to Sclerotinia sclerotiorum. Chin. J. Oil Crop Sci. 2003, 25, 115-116.

32. Yan, X.; Zhang, L.; Chen, B.; Xiong, Z.; Chen, C.; Wang, L.; Yu, J.; Lu, C.; Wei, W. Functional identification and characterization of the Brassica napus transcription factor gene BnAP2, the ortholog of Arabidopsis thaliana APETALA2. PLOS ONE 2012, 7, e33890.

33. Liu, F.; Xiong, X.; Wang, P.; Lei, L.; Zeng, X.; Zhu, L.; Li, Y.; Luo, J.; Fu, D.; Fu, P. Effects of non-procedural factors in Brassica napus genetic transformation. Chin. J. Oil Crop Sci. 2017, 2, 106-121.

34. Yang, L.; Kawakatsu, T.; Wakasa, Y.; Yoine, M.; Takaiwa, F. RNA silencing is induced by the expression of foreign recombinant products in transgenic rice. Plant Sci. 2014, 225, 138-146. [CrossRef] [PubMed]

35. Wang, M.B.; Helliwell, C.A.; Wu, L.M.; Waterhouse, P.M.; Peacock, W.J.; Dennis, E.S. Hairpin RNAs derived from RNA polymerase II and polymerase III promoter-directed transgenes are processed differently in plants. RNA 2008, 14, 903-913. [CrossRef]

36. Dunoyer, P.; Himber, C.; Voinnet, O. DICER-LIKE 4 is required for RNA interference and produces the 21-nucleotide small interfering RNA component of the plant cell-to-cell silencing signal. Nat. Genet. 2005, 37, 1356-1360. [CrossRef]

37. Gasciolli, V.; Mallory, A.C.; Bartel, D.P.; Vaucheret, H. Partially Redundant Functions of Arabidopsis DICER-like Enzymes and a Role for DCL4 in Producing trans-Acting siRNAs. Curr. Biol. 2005, 15, 1494-1500. [CrossRef]

38. Fusaro, A.F.; Matthew, L.; Smith, N.A.; Curtin, S.J.; Dedic-Hagan, J.; Ellacott, G.A.; Watson, J.M.; Wang, M.-B.; Brosnan, C.; Carroll, B.J.; et al. RNA interference-inducing hairpin RNAs in plants act through the viral defence pathway. EMBO Rep. 2006, 7, 1168-1175. [CrossRef]

39. Barnes, W.M. PCR amplification of up to 35-kb DNA with high fidelity and high yield from lambda bacteriophage templates. Proc. Natl. Acad. Sci. USA 1994, 91, 2216-2220. [CrossRef]

40. Jofuku, K.D.; den Boer, B.G.; van Montagu, M.; Okamuro, J.K. Control of Arabidopsis flower and seed development by the homeotic gene APETALA2. Plant Cell 1994, 6, 1211-1225. 
41. Lu, W.; Liu, J.; Xin, Q.; Wan, L.; Hong, D.; Yang, G. A triallelic genetic male sterility locus in Brassica napus: An integrative strategy for its physical mapping and possible local chromosome evolution around it. Ann. Bot. 2013, 111, 305-315. [CrossRef] [PubMed]

42. Xin, Q.; Shen, Y.; Li, X.; Lu, W.; Wang, X.; Han, X.; Dong, F.; Wan, L.; Yang, G.; Hong, D. MS5 mediates early meiotic progression and its natural variants may have applications for hybridproduction in Brassica napus. Plant Cell 2016, 28, 1263-1278. [CrossRef] [PubMed]

43. Lizardi, P.M.; Huang, X.; Zhu, Z.; Bray-Ward, P.; Thomas, D.C.; Ward, D.C. Mutation detection and single-molecule counting using isothermal rolling-circle amplification. Nat. Genet. 1998, 19, 225-232. [CrossRef] [PubMed]

44. Wang, L.; Luo, Y.Z.; Zhang, L.; Jiao, X.M.; Wang, M.B.; Fan, Y.L. Rolling circle amplification-mediated hairpin RNA (RMHR) library construction in plants. Nucleic Acids Res. 2008, 36, e149. [CrossRef] [PubMed]

45. Hirochika, H.; Guiderdoni, E.; An, G.; Hsing, Y.I.; Eun, M.Y.; Han, C.D.; Upadhyaya, N.; Ramachandran, S.; Zhang, Q.; Pereira, A. Rice mutant resources for gene discovery. Plant Mol. Biol. 2004, 54, 325-334. [CrossRef]

46. Fraser, A.G.; Kamath, R.S.; Zipperlen, P.; Martinez-Campos, M.; Sohrmann, M.; Ahringer, J. Functional genomic analysis of C. elegans chromosome I by systematic RNA interference. Nature 2000, 408, 325-330. [CrossRef]

47. Kamath, R.S.; Fraser, A.G.; Dong, Y.; Poulin, G.; Durbin, R.; Gotta, M.; Kanapin, A.; Le Bot, N.; Moreno, S.; Sohrmann, M.; et al. Systematic functional analysis of the Caenorhabditis elegans genome using RNAi. Nature 2003, 421, 231-237. [CrossRef]

48. Wang, L.; Zhao, J.; Fan, Y. Gene cloning and function analysis of ABP9 protein which specifically binds to ABRE2 motif of maize Cat1 gene. Chin. Sci. Bull. 2002, 47, 1871-1875. [CrossRef]

49. De Block, M.; de Brouwer, D.; Tenning, P. Transformation of Brassica napus and Brassica oleracea using Agrobacterium tumefaciens and the expression of the bar and neo genes in the transgenic Plants. Plant Physiol. 1989, 91, 694-701. [CrossRef]

50. Xu, M.Y.; Dong, Y.; Zhang, Q.X.; Zhang, L.; Luo, Y.Z.; Sun, J.; Fan, Y.L.; Wang, L. Identification of miRNAs and their targets from Brassica napus by high-throughput sequencing and degradome analysis. BMC Genom. 2012, 13, 421. [CrossRef] 